\title{
ON A CHARACTERIZATION OF AZUMAYA ALGEBRAS
}

\author{
WARREN DICKS
}

Abstract

A direct proaf of Braun's characterization of Azumaya algebras is given.

Let $A$ be a ring (associative, with 1), $Z$ its centre, and $U=A \otimes_{Z} A^{\text {op }}$. There is an obvious left $U$-action on $A$, which we shall denote by $U \times A \rightarrow A$, $(u, a) \mapsto u * a$. Let $J$ denote the kernel of the map $U \rightarrow A, u \mapsto u * 1$, so $J$ is just the left annihilator of $1 \in A$. Notice $J$ contains $a \otimes 1-1 \otimes a$ for all $a \in A$.

It is well known that the following properties are equivalent, cf.[2, p.52].

(1) $U A$ is a projective generator; that is, there is some $e \in U$ such that $e * 1=1, J e=0, U e U=U$.

(2) $A_{Z}$ is a projective generator and the natural ring homomorphism $U \rightarrow \operatorname{End}_{Z}(A)$ is an isomorphism. $J e=0$.

(3) $U A$ is projective; that is, there is some $e \in U$ such that $e * 1=1$ and

A ring satisfying these equivalent conditions is called an Azumaya algebra. In $[1$, Theorem 4.1], Braun gives the further characterization

(4) There is some $e \in U$ such that $e * 1=1, e * A \subseteq Z$.

In fact (1) $\Leftrightarrow(2)$ by Morita equivalence; (1) $\Rightarrow$ (3) is trivial; and (3) $\Rightarrow(4)$ since $J *(e * A)=(J e) * A=0 * A=0$ so $e * A \subseteq Z$. The purpose of this note is to give a direct proof that $(4) \Rightarrow(1)$.

Thus, suppose there is some $e \in U$ such that $e * 1=1, e * A \subseteq Z$. We show first that $U e U=U$. Let $\mathfrak{a}=\{a \in A \mid a \otimes 1 \in U e U\}$. This is clearly a (two-sided) ideal of $A$; if $\mathfrak{a}=A$ then $U e U=U$ as desired, so we may assume there is a maximal ideal $\mathfrak{m}$ of $A$ containing $a$.

Let us give $U$ two left $U$-module structures $U \times U \rightarrow U,(u, v) \mapsto u *_{1} v, u *_{2} v$ by setting $u *_{1}(a \otimes b)=(u * a) \otimes b, u *_{2}(a \otimes b)=a \otimes\left(u * b_{2}\right)$. It is easy to see these are well-defined. Notice $e *_{2}(a \otimes b)=a \otimes(e * b)=a(e * b) \otimes 1$; notice also that $U *_{2}(U e U) \subseteq U e U$. Hence every element of $e *_{2}(U e U)$ is of the form $a \otimes 1$ for some $a \in \mathfrak{a} \subseteq \mathrm{m}$.

Let $\bar{A}=A / \mathfrak{m}, \bar{Z}=Z /(Z \cap \mathrm{m}), \bar{U}=\bar{A} \otimes_{\bar{Z}} \bar{A}^{\circ p}$ and let the maps $A \rightarrow \bar{A}$, $U \rightarrow \bar{U}$ be denoted $x \mapsto \bar{x}$. Then $\bar{e} * \bar{I}=\overline{1}, \bar{e} * \bar{A} \subseteq \bar{Z}$. But $\bar{Z}$ lies in the centre 
of $\bar{A}$, which in turn is acted on trivially by $\bar{e}$ so $\bar{Z}$ is the centre of $\bar{A}$. Since $\bar{A}$ is simple, it follows that $\bar{U}$ is simple. Also $\bar{e} * \overline{1}=\overline{1}$ so $\bar{e} \neq 0$ and therefore $\bar{U} \bar{e} \bar{U}=\bar{U}$. But $\overline{1 \otimes I}=\bar{e} *_{2}(\overline{1 \otimes 1}) \in \bar{e} *_{2} \bar{U} \bar{e} \bar{U}=\overline{e *_{2} U e U}=0$, a contradiction. This completes the proof that $U e U=U$.

It remains to verify that $J e=0$.

We claim that for any $u \in U,(U e U) *_{2} u \subseteq\left(u *_{1} U\right) U$. Indeed

$$
\begin{gathered}
\left.v e w *_{2}\left(\sum a_{i} \otimes b_{i}\right)=\sum a_{i} \otimes v e w * b_{i}\right)=\sum a_{i} \otimes(v * 1)\left(e w * b_{i}\right) \\
\left.=\sum a_{i}\left(e w * b_{i}\right) \otimes(v * 1)=\sum_{j}\left(\sum_{i} a_{i} \otimes b_{i}\right) *_{1}\left(c_{j} \otimes(v * 1)\right)\right]\left(d_{j} \otimes 1\right)
\end{gathered}
$$

where $e w=\sum c_{j} \otimes d_{j}$. Now $J e * A=J *(e * A) \subseteq J * Z=0$ so $J e * A=0$. But $J e \subseteq U *_{2}(J e)=(U e U) *_{2}(J e) \subseteq\left((J e) *_{1} U\right) U$ which is 0 since $J e * A=0$, so $J e=0$ and (I) holds.

Remarks. (i) The above proof of (3) $\Rightarrow(1)$ is more direct than the one given in [2].

(ii) It is clear from (4) that $A \rightarrow Z, a \mapsto e * a$, is a retraction of $A$ to $Z$; thus in (2) one can strengthen the condition that $A_{Z}$ be a projective generator to $(A / Z)_{Z}$ being finitely generated projective.

\section{References}

1. A. Braun, On Artin's theorem and Azumaya algebras, J. Algebra 77 (1982), 323-332.

2. F. DEMAYER, E. INGRAHAM, Separable algebras over commutative rings, Lecture Notes in Mathematics 181 (1971), Springer-Verlag, New York.

Departament de Matemàtiques Universitat Autònoma de Barcelona 08193 Bellaterra, SPAIN

Rebut el 28 de Gener de 1988 